\title{
Néolithisation du Maroc: Comportements de subsistance et domestication animale
}

\section{Brahim Ouchaou}

\section{(2) OpenEdition}

12 Journals

\section{Édition électronique}

URL : https://journals.openedition.org/encyclopedieberbere/2730

DOI : $10.4000 /$ encyclopedieberbere. 2730

ISSN : 2262-7197

Éditeur

Peeters Publishers

\section{Édition imprimée}

Date de publication : 5 octobre 2012

Pagination : 5512-5520

ISBN : 978-90-429-2718-6

ISSN : 1015-7344

Référence électronique

Brahim Ouchaou, « Néolithisation du Maroc : Comportements de subsistance et domestication animale », Encyclopédie berbère [En ligne], 34 | 2012, document N48, mis en ligne le 15 décembre 2020, consulté le 17 février 2022. URL : http://journals.openedition.org/encyclopedieberbere/2730 ; DOI : https://doi.org/10.4000/encyclopedieberbere.2730

Ce document a été généré automatiquement le 17 février 2022.

(C) Tous droits réservés 


\title{
Néolithisation du Maroc: Comportements de subsistance et domestication animale
}

\author{
Brahim Ouchaou
}

1 La domestication animale représente une composante majeure de la néolithisation (Roubet, N40, N45; Ballouche, N41). Les travaux sur ce thème sont très nombreux (Zeuner 1963, Bökönyi 1974, Gautier 1990, Helmer 1992, Vigne 2011). Schématiquement, on peut distinguer deux courants: dans l'un la domestication des animaux est considérée comme résultat de facteurs externes (insuffisance des ressources, contraintes climatiques, pression démographique, etc.); dans l'autre, elle serait due à des facteurs internes (évolution culturelle, maturations, etc.). Quoi qu'il en soit, un nouveau mode de vie, fondé sur l'économie de production avait fait son apparition durant le $10^{\mathrm{e}}-9^{\mathrm{e}}$ millénaire $\mathrm{BC}$ au Proche Orient. Les voies et modalités de la diffusion de cette transformation sociétale sur le pourtour méditerranéen sont encore peu documentées en Afrique du Nord. Elles sont nettement mieux documentées pour l'Europe, reposant notamment sur les techniques de l'ADN dont la conservation est généralement mauvaise dans les zones à climat chaud (Edwards et al. 2004, Bollongino et al. 2008, Geigl 2008). Leurs résultats ont, en général, confirmé les schémas fondés sur les méthodes classiques.

2 Dans les sites préhistoriques marocains de la frange littorale (Ballouche, Ouchaou \& El Idrissi, N47), la présence du mouton a été mentionnée dès 1936 à El Khenzira par C. Arambourg, mais il s'agit d'une « couche exclusivement mélangée d'éléments récents et subfossiles» (Ruhlman 1936). Pour les faunes d'Achakar, ce paléontologue avait écrit «Sus scrofa domestica est avec Ovis aries l'espèce dominante des niveaux néolithiques des trois grottes» (Arambourg 1967). L'étude des faunes des niveaux supérieurs de quelques sites préhistoriques du Maroc oriental avait montré que les Caprinés (mouton et chèvre) sont dominants et " constituent l'élément de base d'une activité pastorale importante et semblent être associés un peu tardivement aux bovinés » (Wengler et al. 1989). 
3 La grotte de Kaf Taht el-Ghar (Tarradell 1955, Daugas et al. 2008 et 2010, Ramos et al. 2008) est l'un des sites clé pour l'étude de la faune du Néolithique du Maroc. Dès les premiers sondages (1984-1985), il s'est avéré que «l'élevage (chèvre, mouton, boviné) côtoie la céréaliculture attestée par la palynologie » dans une couche datée de $6050 \pm$ 120 B.P., soit 5260 à 4730 av. J.-C. (déterminations de Guadelli in Daugas et al. 1989). Ces résultats sont confirmés par l'étude de la faune des fouilles de 1989 et 1994 (Ouchaou \& Amani 1997, Ouchaou 2000a) qui avait mis l'accent sur la présence d'animaux domestiques depuis les niveaux de la première période d'occupation cardiale et plus précisément dès le sommet de ces niveaux (tab. 1).

Tableau 1. Phases biostratigraphiques de la grotte de Kaf Taht el-Ghar

\begin{tabular}{|c|c|c|c|c|}
\hline \multirow{2}{*}{ Phases } & \multirow{2}{*}{$\begin{array}{l}\text { Principales unités } \\
\text { stratigraphiques }\end{array}$} & \multirow{2}{*}{ Phases culturelles } & \multicolumn{2}{|c|}{$\begin{array}{l}\text { Datations calibrées (calBC) (in Daugas } \\
\text { et al, 2010) }\end{array}$} \\
\hline & & & Référence & Age \\
\hline A & 1001,2001 & Protohistoire & & \\
\hline B & 1002,2002 & Campaniforme & & \\
\hline C & 1003,2003 & $\begin{array}{l}\text { Néolithique moyen / } \\
\text { récent }\end{array}$ & & \\
\hline $\mathrm{D}$ & 1004 & Cardial, période 3 & & \\
\hline E & $1005,1006,1019,2005$ & Cardial, période 2 & Lyon 3821 & $5221-4675$ \\
\hline $\mathrm{F} 1$ & $1020,1021,1030$ & Cardial, période 1 & Rabat 65 & $6227-5669$ \\
\hline F2 & 1025,1028 & Néolithique ancien? & Rabat 66 & $8088-7445$ \\
\hline \multicolumn{5}{|l|}{ Lacune } \\
\hline G & 1029 & Epipaléolithique & Lyon7289 & $14467-13325$ \\
\hline
\end{tabular}

4 Toutefois, le nombre de restes attribués aux animaux domestiques dans la première période d'occupation cardiale est très faible (tableau 2), et ces restes proviennent essentiellement des unités stratigraphiques du sommet de cette phase (US 1020 et 1021), intégrées lors de la dernière sériation (Daugas et al. 2010) à la deuxième période d'occupation cardiale. Dans les autres US $(1005,1019)$ de la deuxième période d'occupation cardiale (phase E), les Caprinés domestiques deviennent nombreux, ce qui permet d'avancer la maîtrise de l'élevage.

Tableau 2. Nombres de restes de quelques groupes zoologiques issus des différents niveaux de Kaf Taht el-Ghar

\begin{tabular}{|l|l|l|l|l|l|l|l|l|}
\hline & A & B & C & D & E & FI & F2 & G \\
\hline Carnivores & 0 & 1 & 14 & 19 & 12 & 41 & 6 & 58 \\
\hline
\end{tabular}




\begin{tabular}{|l|l|l|l|l|l|l|l|l|}
\hline Suides & 59 & 318 & 441 & 492 & 434 & 26 & 3 & 6 \\
\hline Grands ruminants & 28 & 116 & 88 & 142 & 80 & 82 & 9 & 40 \\
\hline Petits ruminants & 247 & 555 & 1383 & 1377 & 831 & 144 & 18 & 865 \\
\hline Caprinés domestiques & 235 & 544 & 1357 & 1306 & 759 & 28 & 0 & 0 \\
\hline Gazelles & 4 & 5 & 13 & 6 & 12 & 23 & 4 & 79 \\
\hline Mouflonà manchettes & 0 & 3 & 11 & 8 & 30 & 60 & 11 & 785 \\
\hline
\end{tabular}

5 Notons que la fragmentation intentionnelle est très importante, mettant en évidence une exploitation optimale des ressources. En effet, sur les 759 restes de Caprines domestiques de la phase $\mathrm{E}$, seulement cinq os longs sont entiers : un métacarpe, un tibia et trois métatarses (fig. 1). En outre, l'on note une dominance des individus jeunes. A titre d'exemple, la phase $\mathrm{E}$ a livré $14 \mathrm{D} 4$ (quatrième molaire lactéale) inférieures (9 droites et 5 gauches) et seulement 5 M3 inférieures (fig. 1).
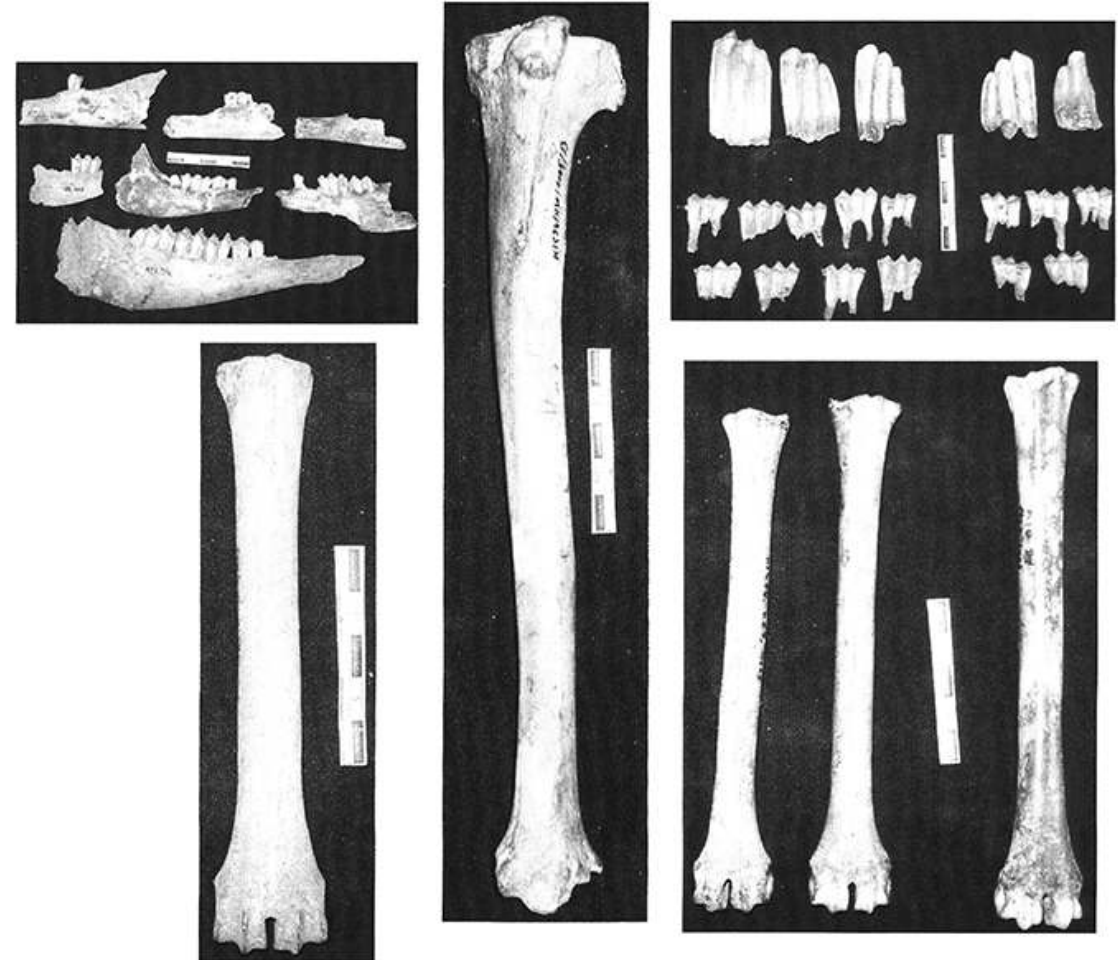

Fig. 1. CAPRINÉS domestiques de LA deUXIÈme PÉRIOde d'occupation CARdiale (PHASE E) dU gISEMENT DE KAF TAHT EL-GHAR.

Cette phase a livré également quelques restes d'un bovin (fig. 2) dont la morphologie et les dimensions sont celles des ossements du bœuf domestique. Bien que le bœuf soit présent dès la phase $E$, ses restes sont très rares durant tout le Néolithique et ne deviennent assez abondants que dans les phases récentes (A et $\mathrm{B})$. 

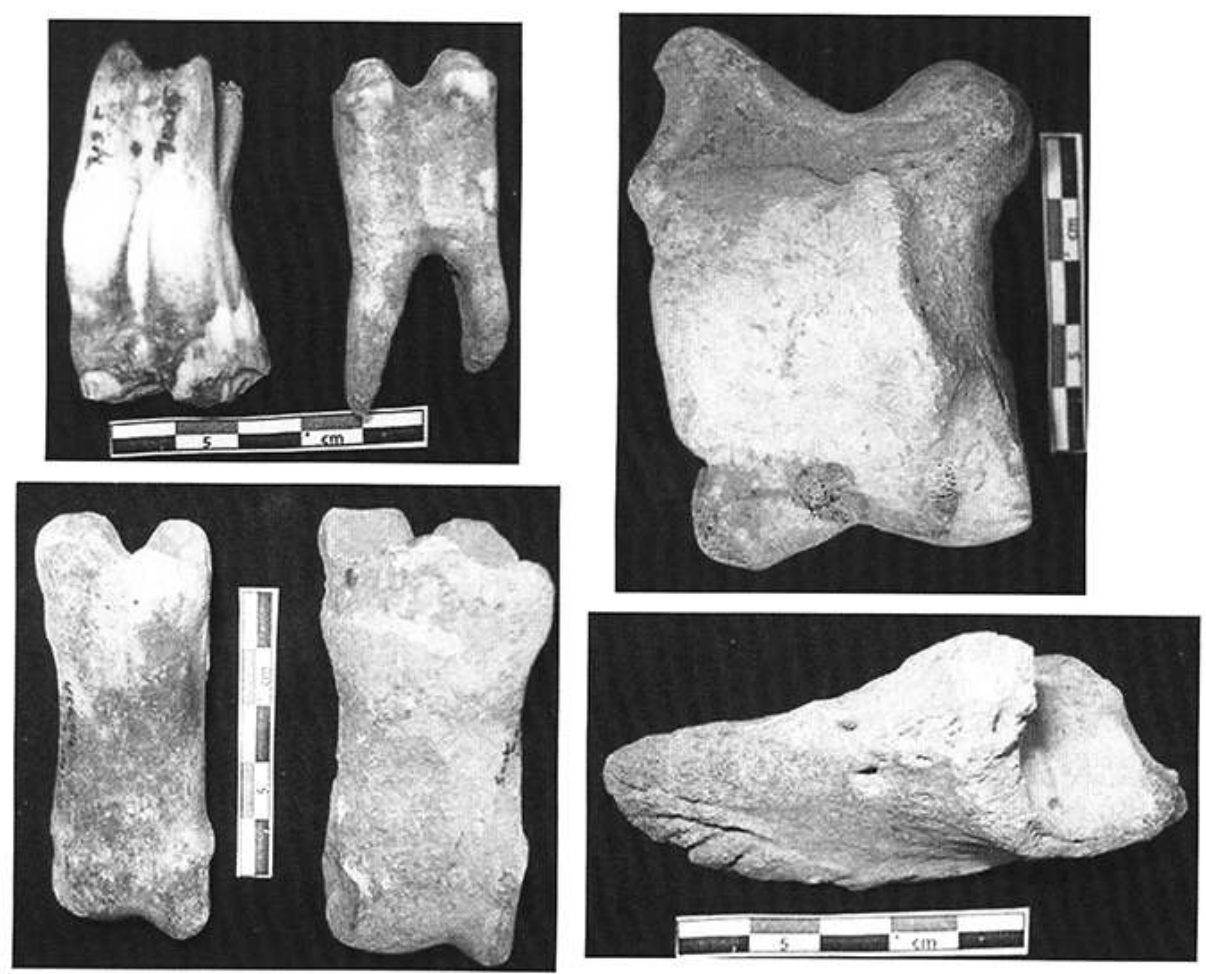

Fig. 2. BœUf DOMESTIQUE de LA DEUXIÈME PÉRIOde d'OCCUPATION CARDIALE (PHASE E) DU gISEMENT DE KAF TAHT EL-GHAR.

7 Les Suidés sont présents dans toutes les phases, mais nettement plus abondants dans les phases où la présence du mouton, chèvre et bœuf est certaine (tableau 2), soit à partir de la phase E (fig. 3).

8 Les comparaisons ostéométriques (Ouchaou 2000a) ont montré qu'il n'y a aucune évolution significative dans la stature de ce suidé de la phase $\mathrm{E}$ (Cardial) à la phase $\mathrm{A}$ (Protohistoire) comme l'illustre l'exemple des astragales (fig. 4). Ces différentes considérations ont amené à considérer ce suidé comme domestique (Ouchaou \& Amani 1997 ; Ouchaou \& Hossini 2008). 

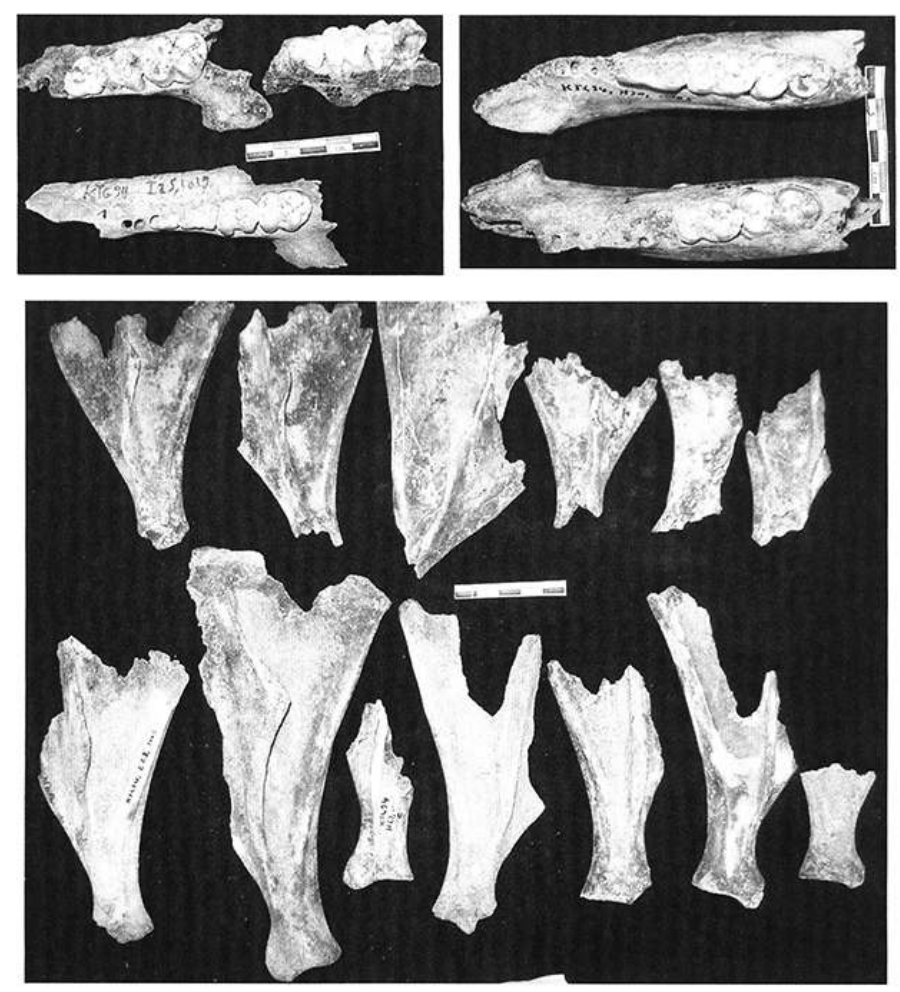

Fig. 3. SUIDÉs dE LA DEUXIÈME PÉRIOdE d'OCCUPATION CARDIALE (PHASE E) DU gISEMENT DE KAF TAHT EL-GHAR.

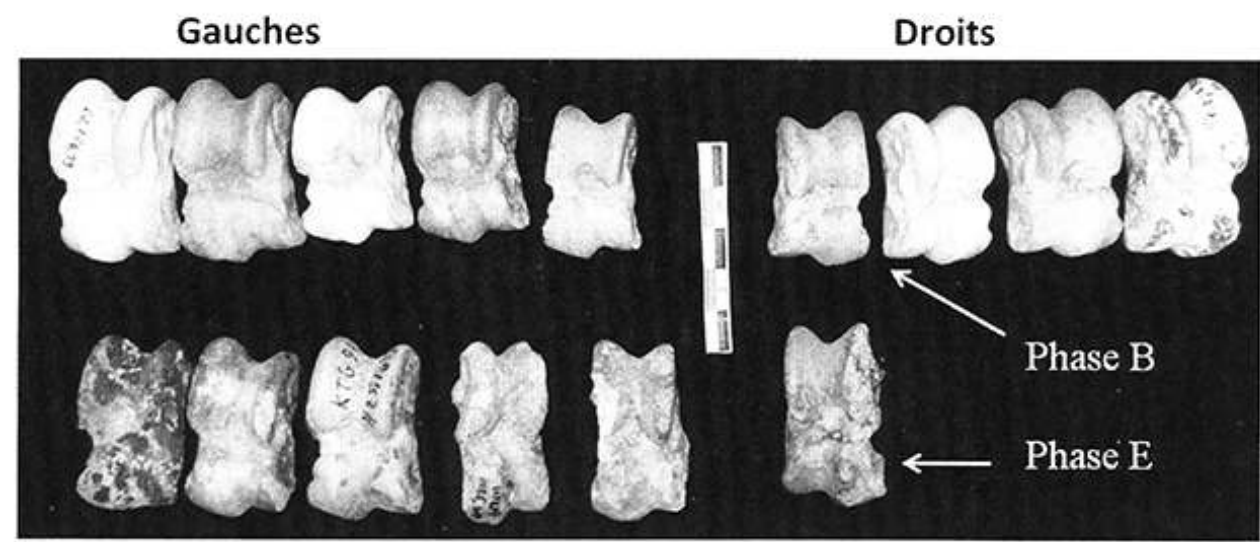

Fig. 4. AStragales des suidés des phases e (CARDial) et B (CAMPANiforme) du gisement de KAF TAHT EL-GHAR.

La présence d'animaux domestiques a été mentionnée dans d'autres sites néolithiques et/ou protohistoriques marocains (tableau 3), notamment Ghar-Khal, Bou-Saria et Kefel-Baroud (Ouchaou 2000b), Hassi Ouenzga et Taghit Haddouch (Ouchaou et al. 2003, Linstädter 2004) et Guenfouda (Aouraghe et al. 2010). Toutefois, dans la majorité des cas, les cadres chronologiques sont imprécis ou inédits. 
Tableau 3. Principaux sites néolithiques et/ou protohistoriques du Maroc ayant livré des restes d'animaux domestiques (1): Arambourg $1967 ;(2)$ : Wengler et al. $1989 ;(3)$ : Ouchaou $2000 ;$ (4) : Ouchaou et al. 2003 ; (5) : Linstädter $2004 ;(6)$ : Aouraghe et al. $2010 ;(7)$ : inédit (détermination Ouchaou).

\begin{tabular}{|l|l|l|l|l|}
\hline Site & Chien & Caprini & Bœuf & Porc \\
\hline Achakar (1) & X & X & ? & X \\
\hline Gisements du Maroc oriental (2) & X & X & X & \\
\hline Kaf Taht el-Ghar (3) & X & X & X & X \\
\hline Kehf-el-Baroud (3) & X & X & X & X \\
\hline Ghar-Khal (3) & X & X & X & X \\
\hline Bou-Saria (3) & X & X & X & X \\
\hline Taghit Haddouch (4) & & $X$ & & \\
\hline Hassi Ouenzga (4,5) & X & X & & \\
\hline Guenfouda (6) & X & X & X & $?$ \\
\hline El Harhoura II (7) & X & X & X & $?$ \\
\hline
\end{tabular}

Il semble que, pour le Maroc septentrional au moins, l'introduction des Caprinés remonte à la fin de la première période d'occupation cardiale. Elle est antérieure à celle $\mathrm{du}$ bœuf, porc et chien, alors qu'ailleurs dans le monde la domestication du chien est, de loin, antérieure à celle des autres animaux. Dès la deuxième période d'occupation cardiale, les Caprinés deviennent abondants et associés aux restes d'un suidé et d'un boviné de petites tailles, attribuables aux formes domestiques (Ouchaou \& Amani 1997, Ouchaou 2000a, Ouchaou \& Hossini 2008). Rappelons que l'attribution des restes de Suidés à la forme domestique sur le seul critère de la taille est critiquée par la majorité des archéozoologues. A partir de la troisième période d'occupation cardiale, les animaux domestiques deviennent largement dominants. La présence du cheval, de l'âne et du chat n'est documentée, par des restes osseux, qu'à partir des niveaux protohistoriques.

11 L'awdad (Ammotragus lervia; cf. "Mouflon », EB XXXII), unique caprin sauvage de la région depuis le Pléistocène supérieur jusqu'à l'Actuel, n'est pas l'ancêtre des Caprini domestiques (Manlius 2010).

De même, «l'hypothèse par laquelle le chacal a parfois été invoqué comme ancêtre possible du chien doit être exclue du fait de sa morphologie dentaire» (Helmer 1992). La rétrogression, dont le rôle est plus important qu'on ne l'admet en général (Gautier 1997) n'est pas sans doute étrangère à la présence, chez les chiens d'Afrique du Nord, de certains caractères qui les rapprochent des chacals.

13 Par conséquent, l'origine allochtone du chien*, du mouton* et de la chèvre*, en absence de souches autochtones possibles, est admise par tous les auteurs. En revanche, l'hypothèse de souches autochtones pour le porc* et le bœuf* a été avancée par 
plusieurs auteurs. Toutefois, jusqu'à présent, aucun site marocain ne peut être considéré comme un site de protodomestication et aucun argument archéozoologique ne permet de confirmer cette hypothèse. En général, la domestication est précédée d'une intensification des rapports entre l'Homme et les formes sauvages ancestrales. Or, en Afrique du Nord, le sanglier et l'aurochs, ancêtres de ces deux formes, n'étaient pas intensivement chassés durant le Paléolithique supérieur. Les gibiers de prédilection étaient le mouflon à manchettes (awdad), les gazelles et l'antilope bubale. De plus, les analyses paléogénétiques ont montré que les animaux domestiques du pourtour méditerranéen, à l'exception du chien peut-être, sont originaires du croissant fertile (Larson et al. 2007, Fernandez et al. 2008, Naderi et al. 2008, Zeder 2008, Vigne 2011). Ce qui permet d'avancer, qu'en l'état actuel des connaissances, aucun argument ne permet d'étayer l'hypothèse d'une origine autochtone pour le porc et le bœuf dans le Néolithique du Maroc.

\section{BIBLIOGRAPHIE}

AOURAGHE H., AGUSTI J., OUCHAOU B., BAILON S., LOPEZ-GARCIA J.M., HADDOUMI H., HAMMOUTI K., OUJAA A. \& BOUGARIANE B., 2010 - “The Holocene vertebrate of Guenfouda, Eastern Morocco”, Historical Biology, $22(1-3)$, p. 320-326.

ARAMBOURG C., 1967 - « Observations sur la faune des grottes d'Hercule près de Tanger, Maroc », in: The Paleolithic of Tangier, Morroco. Excavation at Cap Ashakar 1939-1947, Harvard, Peabody Museum, Bulletin 22, p. 181-186.

BÖKÖNYI S., 1974 - History of domestic Mammals in Central and Eastern Europe, Budapest, Akademi di Kiado, 597 p.

BOLLONGINO R., TRESSET A. \& VIGNE J.-D., 2008 - “Environment and excavation : Pre-lab impacts on ancient DNA analyses", C. R. Palevol, 7, p. 91-98

DAUGAS J.-P., RAYNAl J.-P., Bellouche A., occhiETti S., PICHET P., EVIN J., TEXIER J.-P. \& DÉBENATH A., 1989 - «Le Néolithique nord-atlantique du Maroc : premier essai de chronologie par le radiocarbone », C. R. Acad. Sc., 308, série II, p. 681-687.

DAUGAS J.-P., EL IDRISSI A., BALlOUChE A., MARINVAL P. \& OUCHAOU B., 2008 - « Le Néolithique ancien au Maroc septentrional : données documentaires, sériation typochronologique et hypothèses génétiques », Bulletin de la société préhistorique française, 105 (4), p. 787-812.

DAUGAS J.-P., SBIHI-ALAOUI F.-Z., MIKDAD A., EL IDRISSI A. \& EL GRAOUI M., 2010 - « Le Néolithique du Maroc, 25 ans de coopération franco-marocaine ", Les nouvelles de l'Archéologie, n 120-121, p. 116-121.

EDWARDS C.J., MACHUGH D.E., DOBNEY K.M., MARTIN L., RUSSELL N., HORWITZ L.K., MCINTOSH S.K., MACDONALD K. C., HELMER D., TRESSET A., VIGNE J.-D. \& BRADLEY D. G., 2004 - “Ancient DNA analysis of 101 cattle remains : limits and prospects", Journal of Archaeological Science, 31, p. 695-710. 
FERNANDEZ H., HUGHES S., VIGNE J.-D., HELMER D., HODGINS G., MIQUEL C, HÄNNI C., LUIKART G. \& TABERLET P., 2008 - "Divergent mtDNA lineages of goats in an Early Neolithic sites, far from the initial domestication areas", PNAS, 103 (42), p. 15375-15379.

GAUTIER A., 1990 - La domestication. Et l'Homme créa l'animal, Paris, Errance, Collection des Hespérides, $281 \mathrm{p}$.

GAUTIER A., 1997 - "Once more : the names of domestic animals", Anthropozoologica, 25-26, p. 113-118.

GEIGL E.-M., 2008 - "Palaeogenetics of cattle domestication: Methodological challenges for the study of fossil bones preserved in the domestication centre in Southwest Asia", C. R. Palevol, 7, p. 99-112.

HELMER D., 1992 - La domestication des animaux par les hommes préhistoriques, Paris/Milan, Masson, Collection Préhistoire, 184 p.

LARSON G., ALBARELLA U., DOBNEY K., ROWLEY-CONWY P., SCHIBLER J., TRESSET A., VIGNE J.-P., EDWARDS C. J., SCHLUMBAUM A., DINU A., BĂLĂÇSESCU A., DOL MAN G., TAGLIACOZZO A., MANASERYAN N., PRESTON MIRACLE P. WIJNGAARDEN-BAKKER L. V., MASSETI M., BRADLEY D. G. \& COOPER A., http://www.pnas.org/content/ 104/39/15276 - aff-9 2007 - "Ancient DNA, pig domestication, and the spread of the Neolithic into Europe”, PNAS, 104 (39), p. 15276-15281.

LINSTÄDTER J. 2004 - Zum Frühneolithikum des westlichen Mittelmeerraums. Die Keramik der Fundstelle Hassi Ouenzga, Aachen, Linden soft Verlag EK, 188 p.

MANLIUS N., 2010 - «(M130a) Mouflon (à manchettes) », Encyclopédie berbère, XXXII, p. 5077-5082. NADERI S., REZAEL H.-R., POMPANON F., BLUM M. G. B., NEGRINI R., NAGHASH H.-R., BALKIZ O., MASHKOUR M., GAGGiOTTI O.E., AJMONE-MARSAN P., KENCE A., VIGNE J.-D. \& TABERLET P., 2008 - “The goat domestication process inferred from large-scale mitochondrial DNA analysis of wild and domestic individuals", PNAS, 105 (46), p. 17659-17664

OUCHAOU B., 2000a - Les faunes mammalogiques holocènes des gisements du nord du Maroc : étude paléontologique et observations archéozoologiques. Thèse es-Sciences, Université Moulay Ismaïl, Meknès, $369 \mathrm{p}$.

OUCHAOU B., 2000b - « Les mammifères des gisements néolithiques et protohistoriques du nord du Maroc », Préhistoire Anthropologie Méditerranéennes, 9, p. 73-88.

oUCHAOU B. \& AMANI F., 1997 - « Etude préliminaire des grands Mammifères de Kaf-tah-el-Ghar », Préhistoire Anthropologie Méditerranéennes, 6, p. 53-60.

ouchaou B. \& hossini S. 2008 - "Los restos óseos de Caf taht el Ghar. Imagen de la zoocenosis de la región de Tetuán del Epipaleolítico a la Protohistoria", in : Las ocupaciones humanas de la cueva de Caf taht el Ghar (Tetuán), Ramos J., Zouak M., Bernal D. et Raissouni B., eds., Collection des Monographies du Musée Archéologique de Tétouan, I, p. 35-47.

OUCHAOU B., AMANI F. \& EL MAÂTAOUI M., 2003 - « Liste des espèces de grands mammifères des gisements archéologiques holocènes du nord du Maroc », Notes et Mém. Serv. géol. Maroc, 452, p. 353-356.

RAMOS J., ZOUAK M., BERNAL D. \& RAISSOUNI B., (eds.), 2008 - Las ocupaciones humanas de la cueva de Caf Taht el Ghar (Tetuan), Universidad de Cadiz, Cadiz, 298 p. 
RUHLMAN A., 1936 - Les grottes préhistoriques d' "El Khenzira » (région de Mazagan). Contribution à l'étude du Paléolithique marocain (moyen et supérieur), Publications du Service des Antiquités du Maroc, $130 \mathrm{p}$.

TARADELL M., 1955 - «Avance de la primea campana de excavation en Caf that el Gar », Tamuda, 4, p. 307-325.

VIGNE J.-D., 2011 - "The origins of animal domestication and husbandry : A major change in the history of humanity and the biosphere", C. R. Biologie, 334, p. 171-181.

WENGLER L, DELIBRIAS G., MiCHel P. ET VERNET J.-L., 1989 - « Sites néolithiques du Maroc oriental : cadre chronologique, archéologique et milieu naturel », L’Anthropologie, 93 (4), p. 507-534.

ZEDER M.-A., 2008 - "Domestication and early agriculture in the Mediterranean Basin : Origins, diffusion and impact", PNAS, 105 (33), p. 11597-11604.

ZEUNER F. E., 1963 - A History of domesticated animals, London, Hutchinson, 560 p.

\section{INDEX}

Mots-clés : Alimentation, Maroc, Néolithique 